\title{
Dishevelled controls apical docking and planar polarization of basal bodies in ciliated epithelial cells
}

\author{
Tae Joo Park ${ }^{1}$, Brian J. Mitchell $^{\star}, 2$, Philip B. Abitua ${ }^{\star}, 1$, Chris Kintner ${ }^{2}$, and John B. \\ Wallingford ${ }^{* *}, 1$ \\ ${ }^{1}$ Dept. of Molecular Cell and Developmental Biology \& Institute for Cellular and Molecular Biology \\ University of Texas Austin, Texas 78712 \\ ${ }^{2}$ The Salk Institute for Biological Studies, La Jolla, California 92186, USA
}

\begin{abstract}
The planar cell polarity (PCP) signaling system governs many aspects of polarized cell behavior. Here, we use an in vivo model of vertebrate mucociliary epithelial development to show that Dishevelled (Dvl) is essential for the apical positioning of basal bodies. We find that Dvl and Inturned mediate the activation of the Rho GTPase specifically at basal bodies, and that these three proteins together mediate the docking of basal bodies to the apical plasma membrane. Moreover, we find that the docking involves a Dvl-dependent association of basal bodies with membrane-bound vesicles and with the vesicle-trafficking protein, Sec8. Once docked, Dvl and Rho are once again required for the planar polarization of basal bodies that underlies directional beating of cilia. These results demonstrate novel functions for PCP signaling components and suggest that a common signaling appratus governs both apical docking and planar polarization of basal bodies.
\end{abstract}

\section{Introduction}

Mucociliary epithelia are essential for the proper physiology of many organ systems, and they are composed of two principal cell types, mucus-secreting goblet cells and ciliated cells ${ }^{1-4}$. In the vertebrate airway for example, goblet cells secrete protective mucus, while motile cilia on the neighboring cells beat directionally to clear the mucus away ${ }^{1}$. Defects in cilia structure and function are commonly associated with airway pathologies arising from disease or environmental insults, including asthma, infection, or smoking ${ }^{1}$. Though significant progress has been made recently in understanding the assembly of mono-cilia (reviewed in Refs. 5,6), the molecular mechanisms underlying ciliogenesis in multi-ciliated epithelial cells are only now beginning to receive significant attention ${ }^{7-11}$.

In multi-ciliated cells, basal bodies are replicated deep within the cytoplasm, and their apical movement and docking are thought to involve regulated actin assembly ${ }^{5,12}$ and vesicle trafficking ${ }^{13}$. Indeed, actin is enriched at the apical surface of ciliated epithelial cells ${ }^{7,9}$, and disruption of the actin cytoskeleton blocks basal body migration and ciliogenesis ${ }^{12}$. The assembly of apical actin in multi-ciliated cells involves the Inturned and Fuzzy proteins ${ }^{7}$, as well as the actin regulators ezrin and $\mathrm{RhoA}^{9-11}$. It is not yet clear how these disparate molecules work in concert to promote ciliogenesis.

\footnotetext{
**Author for Correspondence: wallingford@mail.utexas.edu 1 University Station C1000 University of Texas Austin, TX 78712 Phone: 512-232-2784 Fax: 512-471-3878.

*These authors contributed equally to this work.
} 
Another key function of mucociliary epithelia is directional ciliary beating, which is governed by the planar orientation of basal bodies ${ }^{14-16}$. During development of mucociliary epithelia, basal bodies dock apically and cilia are assembled prior to the establishment of planar polarity ${ }^{14,15}$. Planar polarization of ciliary beating has recently been shown to involve a flowmediated positive-feedback mechanism ${ }^{16}$, however molecular regulators of this mechanism have not been identified as yet.

The planar cell polarity (PCP) signaling system is an excellent candidate regulator of ciliary beat polarity ${ }^{17}$. This signaling system controls the actin-dependent planar polarization of structures in a wide range of settings, including Drosophila wing hairs ${ }^{18}$ and the lamellipodia of cells engaged in morphogenetic cell movements ${ }^{19}$. The PCP proteins Inversin/Diego and Vangl2 have been observed near the base of $\mathrm{cilia}^{20,21}$, and others are implicated in mediating cilia morphogenesis by controlling apical actin assembly ${ }^{7,22}$. However, the role of the PCP pathway in directional ciliary beating remains unexplored.

We show here that the core PCP protein Dishevelled (Dvl) plays two important, but separable, roles in multi-ciliated cells. First, Dvl works in concert with the PCP effector Inturned and the Rho GTPase to mediate docking of basal bodies to the apical cell surface. We show that this docking involves a Dvl-dependent association of basal bodies with membrane-bound vesicles and with Sec8, a components of the vesicle-trafficking machinery. Dvl and Rho are also essential for the subsequent planar polarization of basal bodies and for polarized ciliary beating. These results identify the first molecular components of the system by which planar polarization of basal bodies is achieved in a mucociliary epithelium, and they provide a broad framework for understanding the regulation of both apical docking and planar polarization of basal bodies in multi-ciliated cells.

\section{Results}

\section{Dishevelled genes are required for ciliogenesis in multi-ciliated cells}

The epidermis of amphibian embryos is a mucociliary epithelium, a salt-and-pepper mix of mucus-secreting cells and multi-ciliated cells (Fig. 1A, D; Refs. 2,23,24). As in other mucociliary epithelia ${ }^{4,25}$, the ciliated cells are decorated with dozens of very large cilia (Fig. 1B). Several studies demonstrate the morphological, physiological, and molecular similarity between the mucociliary epithelium lining vertebrate airways and that of the Xenopus epidermis ${ }^{2,3,16,24,26}$. All three Xenopus Dvl genes are expressed throughout the epidermis (R. S. Gray, T.J.P., and J.B.W., unpublished). In order to ask if Dvl is involved in ciliogenesis, we designed antisense morpholino-oligonucleotides (MOs) to block translation of Dvl1, Dv12, and Dvl3. We used targeted microinjection to deliver the MOs specifically to the epidermis, thus circumventing the known requirements for Dvl in mesodermal and neural tissues during early development ${ }^{27}$.

Knockdown of any combination of Dvl proteins resulted in significant defects in ciliogenesis (Fig. 1). In embryos injected with Dvl MOs ("morphants"), ciliated cells had significantly fewer cilia and those present were consistently shorter than in controls (Fig. 1c, e). The triple knockdown elicited the most significant defects, but also frequently resulted in embryos that appeared unhealthy, so subsequent experiments were performed with higher doses of a single $\mathrm{MO}$ or with intermediate doses of a double-MO combination. Controls for MO specificity are presented in Supp. Figs. 1 and 2.

To assess the role of Dvl in ciliogenesis, we used 3-dimensional confocal imaging of $\alpha$-tubulin to visualize cilia and actin to visualize the apical cell surface (Fig. 2a-c). When 3D confocal datasets of the ciliated epidermis were viewed in cross section, the cilia can be seen growing exclusively up from the apical cell surface (Fig. 2d, e). In Dvl morphants, far fewer ciliary 
microtubules projected above the apical cell surface (Fig. 2f, g). Microtubules did polymerize in the Dvl morphants, but rather than projecting apically, dense microtubules were observed at or below the apical surface of morphant cells (Fig. 2f, g).

We also observed defects in the actin cytoskeleton in Dvl morphants. Ciliated epidermal cells of normal Xenopus embryos deposit dense actin filaments across the entire apical surface ${ }^{7}$, and this actin mat can be readily observed when 3D confocal datasets are observed in a surface view (Fig. 2k). In this surface view, the individual cilia projecting apically appeared as dots of $\alpha$-tubulin signal (Fig. 2j). Dvl morphants failed to accumulate such apical actin, though actin remained localized at the cell-cell junctions (Fig. $2 \mathrm{~m}$ ). In this surface view, microtubules in morphants were observed as an interwoven bundle at or below the apical cell surface (Fig. 21).

\section{Dishevelled function is required for docking of basal bodies at the apical surface of ciliated cells}

In multi-ciliated cells, basal bodies are formed de novo in the cytoplasm and an actin-based mechanism is thought to transport them apically, where they dock to the plasma membrane ${ }^{5}$. Given the role of Dvl in apical actin assembly in multi-ciliated cells, we assessed the position of basal bodies by $\gamma$-tubulin immunostaining. In control embryos, $\gamma$-tubulin was well-organized and localized tightly to the apical surface of ciliated cells (Fig. 3a). This apical localization of $\gamma$-tubulin was disrupted in Dvl morphants, and instead, the $\gamma$-tubulin signal was observed deep within the cytoplasm (Fig. 3c). To confirm these results, we examined ciliated cells using transmission electron microscopy. Consistent with the immunostaining data, TEM revealed that basal bodies failed to localize apically and failed to dock at the apical membrane in Dvl morphants (Fig. 3e, f). This failure of apical docking did not stem from a general loss of apicobasal polarity in these cells, as apical ZO1 and basolateral Lgl localization were unchanged in Dvl morphants (Supp. Fig. 3 and data not shown). Because all cilia extend from basal bodies, our data suggest that the failure of ciliogenesis in Dvl morphants stems from a failure of basal body docking or maintenance at the apical cell surface, rather than from a failure of cilia assembly.

\section{Dishevelled localizes asymmetrically near the base of cilia}

To better understand the role of Dvl in ciliogenesis, we generated peptide antibodies against Dvl proteins, and an anti-Dvl2 antibody was effective in whole-mount immunostaining. The Dvl2 antibody was concentrated at cell membranes in both ciliated and mucus-secreting cells in the Xenopus ciliated epidermis (Fig. 4a). Moreover, Dvl2 was enriched across the apical surface of ciliated cells, (Fig. 4a-c). Each punctum of Dvl2 signal was observed adjacent to a basal body, as indicated by Centrin 2 coimmunostaining (Fig. 4d). The regular pattern of Dvl2 and Centrin2 localization reflected the planar polarization of basal bodies in mucociliary epithelia (see below). Moreover, Dv12 was consistently observed at the center of the territory labeled by CLAMP-GFP, a marker of the ciliary rootlet (Fig. 4e and see Supp. Fig. 4).

We confirmed this pattern of Dvl localization by expression of a Dv12-GFP fusion protein (Supp. Fig. 5). To determine the regions of the Dvl protein that govern localization to basal bodies, we examined a series of deletion constructs, each fused to GFP (Supp. Fig. 5). We were most interested to observe that a construct containing only the C-terminus of Dvl (Dvl-C1GFP) completely recapitulated the punctate pattern of Dvl localization (Fig. 4f; Supp. Fig. 5b). This C-terminus of Dvl is conserved in Dvl proteins from planaria to humans ${ }^{27}$, and our data suggest that this C-terminus contributes significantly to the localization of Dvl near the base of cilia. 


\section{Expression of the conserved C-terminus of Dishevelled disrupts ciliogenesis}

Dvl is a multi-functional and modular protein, such that its many activities have been effectively dissected by expressing deletion constructs and fragments of the protein ${ }^{19,27-29}$. Because the conserved C-terminus of Dvl localized to basal bodies (Fig. 4f), we overexpressed this fragment specifically in the Xenopus epidermis by targeted mRNA injection. Expression of Dvl-C1 athigher levels severely disrupted normal ciliogenesis. Dvl-C1 expressing cells had fewer microtubules projecting above the apical cell surface (Fig. 2h, i); displayed a loss of apical actin accumulation (Fig. 2o); and failed to localize basal bodies to the apical surface of ciliated cells (Fig. 3c). Moreover, Dvl-C1 elicited the accumulation of interwoven microtubules at and below the apical cell surface (Fig. 2n). Given the localization of Dvl-C1-GFP, we reasoned that this fragment may function as a dominant-negative by associating with structures at the base of cilia and, when overexpressed, may competitively displace wild-type Dvl. Indeed, expression of Dvl-C1 effectively eliminated apical Dvl-GFP foci (Supp. Fig. 6). These data suggest that Dvl localization near the base of cilia, mediated by its C-terminus, plays an essential role in the coordination of ciliogenesis.

\section{Dishevelled mediates Rho activation at the apical surface of ciliated epithelial cells}

Recent data from in vitro culture of mammalian airways suggests that the Rho GTPase is essential for apical docking of basal bodies ${ }^{9}$, and we confirmed this result in the Xenopus epidermis in vivo (Supp. Fig. 7c-e). Because Dvl can associate with and activate Rho (Ref.

${ }^{30}$ ), we considered that Dvl might govern basal body docking by localizing Rho protein or by activating the GTPase activity. We found that GFP-RhoA was enriched at the apical surface of ciliated epithelial cells (Fig. 5a), as in the mammalian airway ${ }^{9}$. However, disruption of Dvl function by knockdown or by expression of Dvl-C1 had no effect on the distribution of RhoAGFP (Fig. 5b and data not shown).

We next assessed the subcellular localization of the activated, GTP-bound form of Rho using an in vivo sensor for Rho activity. rGBD-GFP binds specifically to GTP-bound Rho, and has been used effectively to detect activated Rho in Xenopus embryos using both biochemical and imaging approaches ${ }^{30-32}$. In controls, Rho activation was strongly enriched at the apical surface of ciliated cells (Fig. 5d; Supp. Fig. 7A). Unlike total Rho, active Rho was concentrated in dense foci at the apical surface of ciliated cells (Fig. 5d; Supp. Fig. 7A). These rGBD-GFP foci overlay the foci of co-expressed centrin-RFP, but were larger, suggesting that active Rho decorates the basal body complex (Supp. Fig. 7B). Consistent with a role for Dvl in Rho activation during ciliogenesis, we observed that the number of active Rho foci was greatly reduced when Dvl function was disrupted by knockdown or by expression of Dvl-C1, though Rho activity remained at the cell cortex (Fig. 5e and data not shown). Together, these data indicate that Dvl governs activation, but not localization, of the Rho GTPase during basal body docking in multi-ciliated cells.

\section{Apical localization of Dishevelled and Rho requires the PCP effector protein, Inturned}

The cytoskeletal defects in multi-ciliated cells of Dvl morphants were similar to those resulting from knockdown of the PCP effector, Inturned ${ }^{7}$, prompting us to further explore the mechanism of Inturned function. First, we observed that basal bodies failed to localize to the apical cell surface in Inturned morphants (Fig. 3d). Next, we found that rGBD-GFP foci were missing from the apical surface of ciliated cells lacking Inturned function (Fig. 5f). However, while Rho-GFP localization is normal in Dvl morphants (Fig. 5b), RhoA-GFP was eliminated from the apical cell surface by disruption of Inturned, though RhoA-GFP remained at cell-cell boundaries (Fig. 5c). In Drosophila, Inturned acts only downstream of Dvl ${ }^{18}$, so we were surprised to find that the small, apical foci of Dvl-GFP were largely absent in Inturned morphants (Supp. Fig. 6). This difference in the relationship between Inturned and Dvl in flies and vertebrates may reflect the fact that neither Dvl nor Inturned has been implicated in 
ciliogenesis in Drosophila. Together, these data suggest that although the cilia phenotypes caused by disruption of either Dvl or Inturned are very similar, the underlying mechanism is different: Dvl controls Rho activation but not localization, while Inturned controls the localization of both Rho and of Dvl in multi-ciliated cells.

\section{Dishevelled links to basal body docking to vesicle trafficking and Sec8 localization}

We next sought to understand the cell biological mechanism of Dvl function in basal body docking. Small vesicles are frequently observed in tight association with newly-formed basal bodies in multi-ciliated cells of the mammalian airway ${ }^{13}$ and in cells with primary cilia ${ }^{33}$. It is thought that docking occurs through the fusion of such vesicles to the apical cell surface ${ }^{13}$. Because Dvl, Rho and actin have each been implicated in vesicle or membrane trafficking in varying contexts ${ }^{34-37}$, we examined multi-ciliated cells in Dvl morphants for evidence of membrane trafficking defects. In controls, we observed many basal bodies with tightly associated vesicles (Fig. 6a, inset). In some cases, we appear to have captured basal bodies at just the point when the attached vesicle is fusing to the apical plasma membrane (Supp. Fig. 8A). In contrast, we never observed a tight association between vesicles and basal bodies in Dvl morphants. Rather, the apical cytoplasm of multi-ciliated cells in Dvl morphants was consistently filled with small, isolated vesicles (Fig. 6b; Supp. Fig. 9).

PCP proteins can interact with components of the secretory machinery $34,38,39$, including the exocyst. Because Dvl localizes to basal bodies, we hypothesized that Dvl may facilitate the recruitment of secretory vesicles. We tested this idea by immunostaining for the exocyst component Sec8. In multi-ciliated cells, Sec8 localized to puncta that associated with the basal body marker Centrin-RFP (Fig. 6b-c; Supp. Fig. 8b). By contrast, this association of Sec8 with basal bodies was consistently lost in Dvl morphants. Instead, Sec8 signal was disorganized and often clustered in the cytoplasm apical to the undocked basal bodies in Dvl morphants (Fig. 6e-f; supp. Fig. 8c). Notably, targeting of Sec8 to the apical cell surface does not require a docked basal body; we frequently observed aberrant, Sec8-positive structures projecting apically in Dvl morphants, and such projections were not associated with basal bodies (Supp. Fig. 8d, e). Together, our TEM and immunostaining results suggest that Dvl is required for the orderly association of Sec8-positive vesicles with basal bodies and that this association is in turn required for basal body docking.

\section{Dishevelled and Rho govern both apical docking and planar polarization of basal bodies, and these functions can be experimentally uncoupled}

We next turned our attention the possibility that Dvl governed the planar polarization of basal bodies subsequent to their docking at the apical surface. To assess the planar polarization of basal bodies, we expressed centrin-RFP to mark the basal body and CLAMP-GFP to mark the rootlet (Supp. Fig. 4). Basal bodies in control cells were obviously aligned along their long axes (Fig. 7a). This polarity was quantified with a circular plot of the angular orientation of individual basal bodies. All basal bodies in each control cell had similar angular measurements; the circular standard deviation was quite low, 21 (Fig. 7c and see Ref. 16). We confirmed this result by examining the pattern of Mig12-GFP, which also marks an elongate structure at the base of cilia, and the ordered alignment of these structures reflected the planar polarization observed with Centrin/CLAMP (Supp. Fig. 10 and see ${ }^{\text {Ref. }}{ }^{2}$ ).

Because basal bodies fail to dock in Dvl morphants, we again turned to deletion constructs of Dvl to dissect the protein's function ${ }^{19,27-30}$. When overexpressed in the epidermis, the welldescribed Xdd1 deletion ${ }^{19,29,30}$ had only modest effects on ciliogenesis, even at very high doses (see below, Fig. 8f, g and Supp Movies 5 \& 7). Despite the normal appearance of cilia however, Xdd1 expression severely impaired the planar polarization of basal bodies. Failure 
of basal body alignment was observed in Xdd1 expressing embryos using either centrin-RFP/ CLAMP-GFP (Fig. 7b, d; CSD = 62) or using Mig12-GFP (CSD = 101; Supp. Fig. 10).

Finally, we found that, at the doses used, many embryos expressing dominant-negative RhoA successfully completed ciliogenesis. The effect of RhoA-N17 is dose dependent, but higher doses were embryotoxic. We took advantage of this finding to assess the role of the Rho GTPase in planar polarization of basal bodies. Consistent with a role for Rho in planar polarization, we found that docked basal bodies failed to align in RhoA-N19 expressing cells (CSD = 76; Supp. Fig. 10).

\section{Dishevelled and Rho are required for polarized ciliary beating}

The planar polarity of basal bodies underlies polarized beating and the generation of directional fluid flow across ciliated epithelia ${ }^{14,16}$. Based upon the disruption of basal body planar polarization, we speculated that such flow should be disrupted by expression of Xdd1 or RhoN19. We therefore monitored fluid flow across the Xenopus epidermis by adding latex beads to the culture media and making time-lapse movies of bead movement. In control embryos, beads moved quickly and in a well-organized manner (Fig. 8a; Movie 1), as previously described ${ }^{26}$. By contrast, in Xdd1 or RhoA-N19 injected embryos, we frequently observed an unorganized flow of beads (Fig. 8b-d; Movies $2 \& 3$ ). In many locations, we observed beads that were swirling and turning (Fig. 8b, c). We also observed in experimental embryos, that beads in the same region were moving in different directions (Fig. 8b, c; Movies $2 \& 3$ ).

Ciliary motility is required for the establishment of polarized beating ${ }^{16}$, so it was possible that the reduced directionality of fluid flow in experimental embryos resulted from disrupted cilia motility. To assess this issue, we used high-speed confocal imaging of cilia labeled with tauGFP. Overall, beat frequency appeared comparable between controls and Xdd1 expressing embryos (Fig. 8f, g; Movies 4-7), though modest reductions in beat frequency were observed in a few Xdd1-expressing cells (Movie 7, cells on left). Ciliary beating appeared quite disorganized in many Xdd1 expressing cells as compared to controls (Fig. 8f, g; Movies 4-7). In sum, the orientation of basal bodies, the direction of fluid flow across the epithelium, and the time-lapse analysis of ciliary beating indicate a key role for Dvl and Rho in the planar polarization of ciliary beating in a mucociliary epithelium.

\section{Discussion}

The motile cilia of multi-ciliated cells are central to the physiology of a variety of organ systems, in particular the airway. In this report, we use an in vivo model of mucociliary epithelial development ${ }^{2}$ to provide evidence that a single regulatory network governs not only cilia morphogenesis but also polarized ciliary beating. First, Dvl is required for actin assembly and basal body docking to the apical cell surface in multi-ciliated cells. Dvl may also be required for maintenance of basal bodies at the apical surface. We trace this function to a requirement for Dvl, together with the effector protein Inturned, for local activation of the Rho GTPase, specifically at the apical surface of ciliated epithelial cells. Moreover, we show that Dvl links basal body docking to vesicle traffic and Sec8 localization. Together, our data suggest that Dvl, Inturned, Rho and Sec8 comprise a core framework for regulating the docking and/or maintenance of basal bodies to the apical plasma membrane in multi-ciliated cells.

TEM studies of both multi-ciliated cells and cells with only a primary cilium have suggested that basal body docking is achieved by association of basal bodies with cytoplasmic vesicles which then fuse to the apical cell surface by a mechanism similar to exocytosis ${ }^{13,33}$. It is possible then to envision a model whereby Dvl associates closely with the basal body complex, and facilitates the recruitment of Sec8-positive exocytic vesicles. In this model, Inturned may serve as a scaffold which places Rho in proximity with Dvl, thereby activating the 
GTPase $^{30,32}$. Because actin is crucial for fusion of exocytic vesicles to the plasma membrane ${ }^{37}$, it is noteworthy that apical actin assembly is also disrupted in multi-ciliated cells lacking Dvl or Inturned. While Rho and Dvl activate the formin protein Daam1 during gastrulation ${ }^{30}$, we found no evidence of a role for Daam1 in ciliogenesis (not shown). Dvl probably governs actin assembly in multi-ciliated cells via CapZIP, which is phosphorylated downstream of Dishevelled and governs actin assembly and ciliogenesis of motile mono-cilia in zebrafish embryos ${ }^{22}$.

While the detailed mechanisms of Dvl function in basal body docking remain to be elucidated, this link to vesicle traffic has broad implications. In addition to basal body docking, Dvl controls canonical Wnt signaling, and PCP signaling, but a unifying mechanism for Dvl function has yet to be established ${ }^{27}$. Our data suggest that governance of membrane traffic may be a universal function for Dvl. A direct association between Dvl and membranous vesicles is a matter of ongoing debate (e.g. ${ }^{40}$ ), however several lines of evidence support this hypothesis. First, association of Dvl with membrane-bound vesicles has been shown to be important for canonical Wnt signaling ${ }^{36}$, and it facilitates the aggregation of the Wnt co-receptor Lrp6 into membrane-associated signalsomes ${ }^{35}$. Second, intracellular PCP signaling components, including Dvl, interact functionally and physically with components of the membrane trafficking machinery $34,38,39,41$. Finally, Dvl has been implicated in endo- and exocytosis at synapses $^{39,42}$.

A connection between Dvl, membrane traffic, and the basal body is particularly intriguing in light of recent results linking cilia and Wnt signaling. While the present work explores the role of Dvl upstream of cilia formation, other recent studies have shown the converse: that the cilium or basal body are essential organelles governing the activity of Wnt signaling pathways ${ }^{20,21 \text {, }}$ 43. The interconnections between Wnt signaling, vesicle traffic, and ciliogenesis are perhaps best exemplified by the BBS4 protein. BBS4 interacts genetically with both canonical Wnt and PCP signaling ${ }^{21,43}$, but the protein also mediates membrane delivery to the primary cilium and controls microtubule organization and ciliogenesis ${ }^{44,45}$. Moreover, ciliary and basal body proteins (including BBS4) can directly regulate Dvl phosphorylation, localization, and stability ${ }^{20,43}$. Connecting these disparate findings will be a challenge for future studies, but our data suggest that Dvl's influence on membrane traffic may be a central facet of this protein's function.

Finally, a connection between Dvl, membrane traffic and basal body docking may have implications for understanding the evolution of ciliated cells. It has been suggested that the eukaryotic cilium evolved initially from a specialized apparatus, related to the COPI-clathrin complex, that controlled local delivery of vesicles to the cell membrane ${ }^{46}$. Moreover, a recent study found that the Bardet-Beidel Syndrome proteins function together with Rab8 to coordinate the delivery of ciliary membrane ${ }^{45}$, and it seems possible then that the exocyst complex might have been similarly co-opted during evolution for the specialized purpose of docking basal bodies.

It is also interesting that both Dvl and Rho play roles in apical docking that were experimentally separable from those involved in planar polarization of basal bodies. Because the direction of ciliary beat is established subsequent to basal body docking and cilia outgrowth ${ }^{14-16}$, it seems reasonable to conclude that Dvl and Rho act first in apical docking and only later in planar polarization. Nonetheless, it will be important now to understand how Dvl at the basal body discriminates between its apical docking function and its planar polarity function. One possibility is that Rho activity for apical docking and for planar polarization are regulated by distinct guanine-nucleotide exchange factors. This idea is supported by the finding that knockdown of ARHGEF11 causes a spectrum of embryonic defects that suggest defective cilia-mediated fluid flow ${ }^{47,48}$. In ARHGEF11 morphants, apical actin is disrupted in ciliated 
cells, but cilia are grossly normal and motile ${ }^{47}$, so this GEF may function exclusively in polarization of ciliary beat.

In conclusion, we have shown here that Dishevelled, by controlling both apical docking and planar polarization of basal bodies, lies at the nexus of cilia morphogenesis and cilia function. Cilia are widely recognized to play important roles in development and disease ${ }^{1,6,17}$, so it is noteworthy that polymorphisms in at least one PCP gene have been associated recently with neural tube defects in humans ${ }^{49}$. Our data suggest that this pathway also impacts directed ciliary beat, so such polymorphisms may also be complicating factors in asthma or other airway pathologies that are impacted by mucociliary dysfunction ${ }^{1}$. The results presented here provide the foundation for more detailed elucidation of these important genetic systems, and such studies should impact our understanding of mucociliary epithelia and the pathologies that affect them.

\section{Experimental Procedures}

\section{Embryo manipulations}

Female adult Xenopus laevis were ovulated by injection of human chorionic gonadotropin and eggs were fertilized in vitro and dejellied in $3 \%$ cysteine $(\mathrm{pH} 7.9)$ and subsequently reared in $1 / 3 x$ MMR. For microinjections, embryos were placed in a solution of $2.5 \%$ ficoll in $1 / 3 x$ MMR, and injected using forceps and an Oxford universal micromanipulator, then reared in $2 \%$ ficoll $+1 / 3 \times$ MMR to stage 9 , then washed and reared in 1/3x MMR alone. To analyze epidermal cilia formation, embryos were fixed between stage 25 to 27 and immunostained. Embryo culture, solutions, in vitro transcription, and in situ hybridization were performed using standard methods. For all experiments, MOs were injected at 20-60ng/blastomeres and various amount of mRNAs were injected. For most experiments, injections were made into the two ventral cells at the four-cell stage to target ciliated epidermis.

For assessment of fluid flow, embryos were immobilized in modeling clay and small latex beads were added to the culture media. Time-lapse microscopy was then performed with a Zeiss DiscoveryV12 stereoscope, a Spot digital camera, and ImageProPlus software.

\section{Cloning and Morpholino oligo design}

The C-terminus of Xenopus Dvl2, spanning amino acid 501-736, was amplified by PCR and cloned into CS107-3STOP or CS107-GFP-3STOP. Mig-12 cDNA was obtained from NIBB (Clone I.D. X1034116). The ORF of Mig-12 was amplified by PCR and cloned into pCS107GFP-3STOP. Xenopus Centrin2(Unigene ID: X1.50473, basal body marker) and CLAMP (Unigene ID: X1.26316, rootlet marker, see Supp. Fig. 4) were cloned to pCS2+ and fused Cterminally to GFP or RFP. Dvl1(Image clone ID: 6956452), Dvl2(Image clone ID: 6868624) and Dvl3(NIBB ID: Xl190g16) cDNAs were obtained from NIBB or I.M.A.G.E consortium and subcloned in CS107-3STOP for in vitro translation. Translation blocking antisense morpholinos for Dvl isoforms were designed based on the sequences from NIBB database (Dvl-1, CD362087(ATCC); Dvl-2 (previously Xdsh); $D v l-3$, XL190g16 (NIBB)). MOs were obtained from Gene Tools:

MO sequence for Inturned was previously reported ${ }^{7}$.

\section{Western blotting and in vitro translation}

Embryos were injected with MOs and lysed in M-PER Mammalian Protein Extraction Reagent (Pierce) containing protease inhibitor cocktail(Sigma) and analyzed by SDS-PAGE and Western blot analysis. For in vitro translation, cDNAs were mixed with MOs and in vitro translation reaction were performed in the presence of biotinylated lysine according to 
manufacture's manual (TNT® Quick Coupled Transcription/Translation Systems, Transcend ${ }^{\mathrm{TM}}$ Non-Radioactive Translation Detection Systems, Promega). The reaction mixtures were analyzed by SDS-PAGE and Western blotting probed using HRP-conjugated Streptavidin.

\section{Immunostaining and Confocal imaging and analysis}

For epidermal staining, embryos were fixed in MEMFA at stage 25 to 27 and washed with PBST (PBS+0.1\% TritonX-100). The embryos were blocked for $30 \mathrm{~min}$ in 10\% FBS and 5\% DMSO in PBST. The embryos were incubated with a primary antibody in the blocking solution at $4{ }^{\circ} \mathrm{C}$ overnight. After washing with PBST for 30min three times, the embryos were incubated with Alexa Flour 555 goat anti-mouse IgG or Alexa Flour 488 goat anti-mouse IgG in the blocking solution at $4^{\circ} \mathrm{C}$ overnight followed by washing in PBST for 30min three times. In all cases, embryos were mounted in PPST and 3D projections of cilia were made by collecting overlapping sections with a Zeiss LSM5 PASCAL confocal microscope. Anti $\alpha$-tubulin antibody(T9026) was purchased from Sigma. Anti- $\gamma$-tubulin(ab27076), Sec8(ab13254), and Centrin(ab11257) antibodies were purchased from Abcam. Anti-Zo1 antibody(61-7300) was purchased from Zymed Laboratories. Dvl2 and Dvl3 antibodies were raised in rabbits against peptide sequences below by Open Biosystems.

\section{Dvl2: SVISMRRDRVRRRESS}

\section{Dvl3: NRSSTEKRKDRETKGGDSKS}

High-speed confocal imaging was performed by time-lapse collection of single optical sections at a frame rate of 360fps using a Zeiss 5LIVE microscope. Images were collected from intact, living embryos expressing tau-GFP. 3D projections, image processing, and image analysis were performed with LSM5, Image ProPlus, and Adobe Photoshop software. For the circular analysis of basal body polarity, statistiXL 1.7 program was used.

\section{Electron microscopy}

Electron microscopy was performed roughly according to Ref 3 . Specimens for electron microscopy were fixed in a phosphate buffered $3 \%$ glutaraldehyde and then post-fixed in a phosphate buffered $2 \%$ osmium tetroxide. Tissue was then dehydrated in a stepwise manner. For transmission electron microscopy, the embryos were embedded in Araldite 502 resin and $50 \mathrm{~nm}$ sections were cut using an ultramicrotome. Scanning electron microscopy specimens were critical point dried and then mounted on stubs using silver paste. Afterwards they were sputter coated with $20 \mathrm{~nm}$ of platinum.

\section{Supplementary Material}

Refer to Web version on PubMed Central for supplementary material.

\section{Acknowledgments}

We thank R. Gray, J. Hayes and E. Herrington for technical assistance and The Institute for Cellular and Molecular Biology at UT, Austin Core Facility for help with electron microscopy and J. Kirk with Zeiss Microimaging for help with high-speed confocal imaging. We thank A. Ewald for critical reading and discussion. T.J.P. is supported by the William S. Livingston Graduate Fellowship from UT, Austin. This work was supported by NIH/NIGMS grants to J.B.W. and C.K. and by grants to J.B.W. from The Sandler Program for Asthma Research, The Burroughs-Wellcome Fund, and The March of Dimes.

\section{References}

1. Chilvers MA, O'Callaghan C. Local mucociliary defence mechanisms. Paediatr Respir Rev 2000;1:2734. [PubMed: 16263440] 
2. Hayes JM, et al. Identification of novel ciliogenesis factors using a new in vivo model for mucociliary epithelial development. Dev Biol 2007;312:115-30. [PubMed: 17961536]

3. Steinman RM. An electron microscopic study of ciliogenesis in developing epidermis and trachea in the embryo of Xenopus laevis. Am J Anat 1968;122:19-55. [PubMed: 5654501]

4. Raji AR, Naserpour M. Light and Electron Microscopic Studies of the Trachea in the One-Humped Camel (Camelus dromedarius). Anat Histol Embryol 2007;36:10-3. [PubMed: 17266660]

5. Dawe HR, Farr H, Gull K. Centriole/basal body morphogenesis and migration during ciliogenesis in animal cells. J Cell Sci 2007;120:7-15. [PubMed: 17182899]

6. Scholey JM, Anderson KV. Intraflagellar transport and cilium-based signaling. Cell 2006;125:43942. [PubMed: 16678091]

7. Park TJ, Haigo SL, Wallingford JB. Ciliogenesis defects in embryos lacking inturned or fuzzy function are associated with failure of planar cell polarity and Hedgehog signaling. Nat Genet 2006;38:30311. [PubMed: 16493421]

8. Vladar EK, Stearns T. Molecular characterization of centriole assembly in ciliated epithelial cells. J Cell Biol 2007;178:31-42. [PubMed: 17606865]

9. Pan J, You Y, Huang T, Brody SL. RhoA-mediated apical actin enrichment is required for ciliogenesis and promoted by Foxj1. J Cell Sci 2007;120:1868-76. [PubMed: 17488776]

10. Huang T, et al. Foxj1 is required for apical localization of ezrin in airway epithelial cells. J Cell Sci 2003;116:4935-45. [PubMed: 14625387]

11. Gomperts BN, Gong-Cooper X, Hackett BP. Foxj1 regulates basal body anchoring to the cytoskeleton of ciliated pulmonary epithelial cells. J Cell Sci 2004;117:1329-37. [PubMed: 14996907]

12. Boisvieux-Ulrich E, Laine MC, Sandoz D. Cytochalasin D inhibits basal body migration and ciliary elongation in quail oviduct epithelium. Cell Tissue Res 1990;259:443-54. [PubMed: 2317839]

13. Sorokin SP. Reconstructions of centriole formation and ciliogenesis in mammalian lungs. J Cell Sci 1968;3:207-30. [PubMed: 5661997]

14. Boisvieux-Ulrich E, Laine MC, Sandoz D. The orientation of ciliary basal bodies in quail oviduct is related to the ciliary beating cycle commencement. Biol Cell 1985;55:147-50. [PubMed: 2937490]

15. Frisch D, Farbman AI. Development of order during ciliogenesis. Anat Rec 1968;162:221-32. [PubMed: 5726142]

16. Mitchell B, Jacobs R, Li J, Chien S, Kintner C. A positive feedback mechanism governs the polarity and motion of motile cilia. Nature 2007;447:97-101. [PubMed: 17450123]

17. Wallingford JB. Planar cell polarity, ciliogenesis and neural tube defects. Hum Mol Genet 2006;15 (Spec No 2):R227-34. [PubMed: 16987888]

18. Adler PN, Zhu C, Stone D. Inturned Localizes to the Proximal Side of Wing Cells under the Instruction of Upstream Planar Polarity Proteins. Curr Biol 2004;14:2046-51. [PubMed: 15556868]

19. Wallingford JB, et al. Dishevelled controls cell polarity during Xenopus gastrulation. Nature 2000;405:81-85. [PubMed: 10811222]

20. Simons M, et al. Inversin, the gene product mutated in nephronophthisis type II, functions as a molecular switch between Wnt signaling pathways. Nat Genet 2005;37:537-43. [PubMed: 15852005]

21. Ross AJ, et al. Disruption of Bardet-Biedl syndrome ciliary proteins perturbs planar cell polarity in vertebrates. Nat Genet 2005;37:1135-40. [PubMed: 16170314]

22. Oishi I, Kawakami Y, Raya A, Callol-Massot C, Izpisua Belmonte JC. Regulation of primary cilia formation and left-right patterning in zebrafish by a noncanonical Wnt signaling mediator, duboraya. Nat Genet 2006;38:1316-22. [PubMed: 17013396]

23. Assheton R. Notes on the ciliation of the ectoderm of the amphibian embryo. Q. J. Microsc. Sci 1896;38:465-484.

24. Nokhbatolfoghahai M, Downie JR, Clelland AK, Rennison K. The surface ciliation of anuran amphibian embryos and early larvae: Patterns, timing differences and functions. Journal of Natural History 2005;39:887-929.

25. Toskala E, Smiley-Jewell SM, Wong VJ, King D, Plopper CG. Temporal and spatial distribution of ciliogenesis in the tracheobronchial airways of mice. Am J Physiol Lung Cell Mol Physiol 2005;289:L454-9. [PubMed: 15879461] 
26. Konig G, Hausen P. Planar polarity in the ciliated epidermis of Xenopus embryos. Dev Biol 1993;160:355-68. [PubMed: 8253269]

27. Wallingford JB, Habas R. The developmental biology of Dishevelled: an enigmatic protein governing cell fate and cell polarity. Development 2005;132:4421-36. [PubMed: 16192308]

28. Axelrod JD, Miller JR, Shulman JM, Moon RT, Perrimon N. Differential recruitment of Dishevelled provides signaling specificity in the planar cell polarity and Wingless signaling pathways. Genes Dev 1998;12:2610-22. [PubMed: 9716412]

29. Rothbächer U, et al. Dishevelled phosphorylation, subcellular localization and homomerization regulate its role in early embryogenesis. EMBO J 2000;19:1010-1022. [PubMed: 10698942]

30. Habas R, Kato Y, He X. Wnt/Frizzled activation of Rho regulates vertebrate gastrulation and requires a novel Formin homology protein Daam1. Cell 2001;107:843-54. [PubMed: 11779461]

31. Benink HA, Bement WM. Concentric zones of active RhoA and Cdc42 around single cell wounds. J Cell Biol 2005;168:429-39. [PubMed: 15684032]

32. Park TJ, Gray RS, Sato A, Habas R, Wallingford JB. Subcellular localization and signaling properties of dishevelled in developing vertebrate embryos. Curr Biol 2005;15:1039-44. [PubMed: 15936275]

33. Cohen E, Binet S, Meininger V. Ciliogenesis and centriole formation in the mouse embryonic nervous system. An ultrastructural analysis. Biol Cell 1988;62:165-9. [PubMed: 3390625]

34. Yu A, et al. Association of Dishevelled with the clathrin AP-2 adaptor is required for Frizzled endocytosis and planar cell polarity signaling. Dev Cell 2007;12:129-41. [PubMed: 17199046]

35. Bilic J, et al. Wnt induces LRP6 signalosomes and promotes dishevelled-dependent LRP6 phosphorylation. Science 2007;316:1619-22. [PubMed: 17569865]

36. Capelluto DG, et al. The DIX domain targets dishevelled to actin stress fibres and vesicular membranes. Nature 2002;419:726-9. [PubMed: 12384700]

37. Lanzetti L. Actin in membrane trafficking. Curr Opin Cell Biol 2007;19:453-8. [PubMed: 17616384]

38. Classen AK, Anderson KI, Marois E, Eaton S. Hexagonal packing of Drosophila wing epithelial cells by the planar cell polarity pathway. Dev Cell 2005;9:805-17. [PubMed: 16326392]

39. Kishida $S$, et al. Dvl regulates endo- and exocytotic processes through binding to synaptotagmin. Genes Cells 2007;12:49-61. [PubMed: 17212654]

40. Schwarz-Romond T, Merrifield C, Nichols BJ, Bienz M. The Wnt signalling effector Dishevelled forms dynamic protein assemblies rather than stable associations with cytoplasmic vesicles. J Cell Sci 2005;118:5269-77. [PubMed: 16263762]

41. Chen W, et al. Dishevelled 2 recruits beta-arrestin 2 to mediate Wnt5A-stimulated endocytosis of Frizzled 4. Science 2003;301:1391-4. [PubMed: 12958364]

42. Ahmad-Annuar A, et al. Signaling across the synapse: a role for Wnt and Dishevelled in presynaptic assembly and neurotransmitter release. J Cell Biol 2006;174:127-39. [PubMed: 16818724]

43. Gerdes JM, et al. Disruption of the basal body compromises proteasomal function and perturbs intracellular Wnt response. Nat Genet 2007;39:1350-60. [PubMed: 17906624]

44. Kim JC, et al. The Bardet-Biedl protein BBS4 targets cargo to the pericentriolar region and is required for microtubule anchoring and cell cycle progression. Nat Genet 2004;36:462-70. [PubMed: 15107855]

45. Nachury MV, et al. A core complex of BBS proteins cooperates with the GTPase Rab8 to promote ciliary membrane biogenesis. Cell 2007;129:1201-13. [PubMed: 17574030]

46. Jekely G, Arendt D. Evolution of intraflagellar transport from coated vesicles and autogenous origin of the eukaryotic cilium. Bioessays 2006;28:191-8. [PubMed: 16435301]

47. Panizzi JR, Jessen JR, Drummond IA, Solnica-Krezel L. New functions for a vertebrate Rho guanine nucleotide exchange factor in ciliated epithelia. Development 2007;134:921-31. [PubMed: 17267448]

48. Kramer-Zucker AG, et al. Cilia-driven fluid flow in the zebrafish pronephros, brain and Kupffer's vesicle is required for normal organogenesis. Development 2005;132:1907-21. [PubMed: 15790966]

49. Kibar Z, et al. Mutations in VANGL1 associated with neural-tube defects. N Engl J Med 2007;356:1432-7. [PubMed: 17409324] 

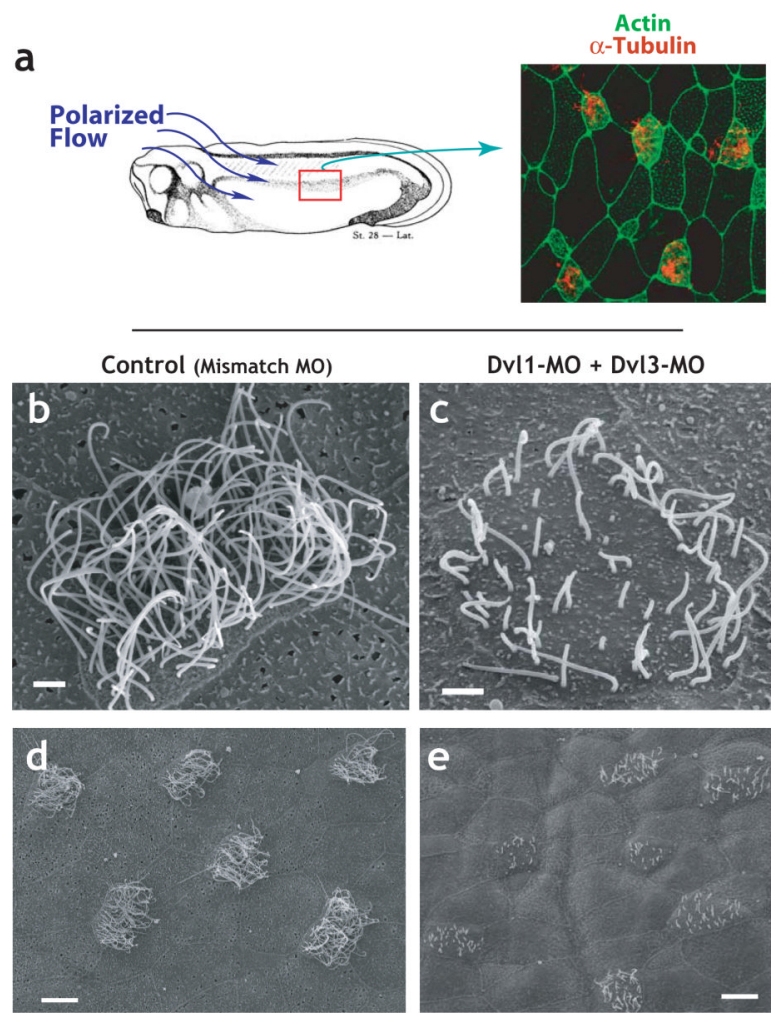

Figure 1. Dishevelled is essential for ciliogenesis in multi-ciliated cells

A. The epidermis of the Xenopus embryo is composed of multi-ciliated cells and mucus secreting cells Ciliated cells, labelled by $\alpha$-tubulin immunostaining (red), are evenly-spaced between mucus-secrteing cells ${ }^{2}$. Polarized beating of cilia generates directional flow across the epithelium ${ }^{16,26}$. B. riposte2ormal ciliated cell from a control-injected embryo (injected with a mismatched morpholino-oligonucleotide). C. Defective cilia outgrowth in Dvl1/Dvl3 double morphants. D. Wider field view of control (mismatch-MO injected) embryo. E. Wider field view of Dvl1/Dvl3 double morphant embryo showing many affected cells. 

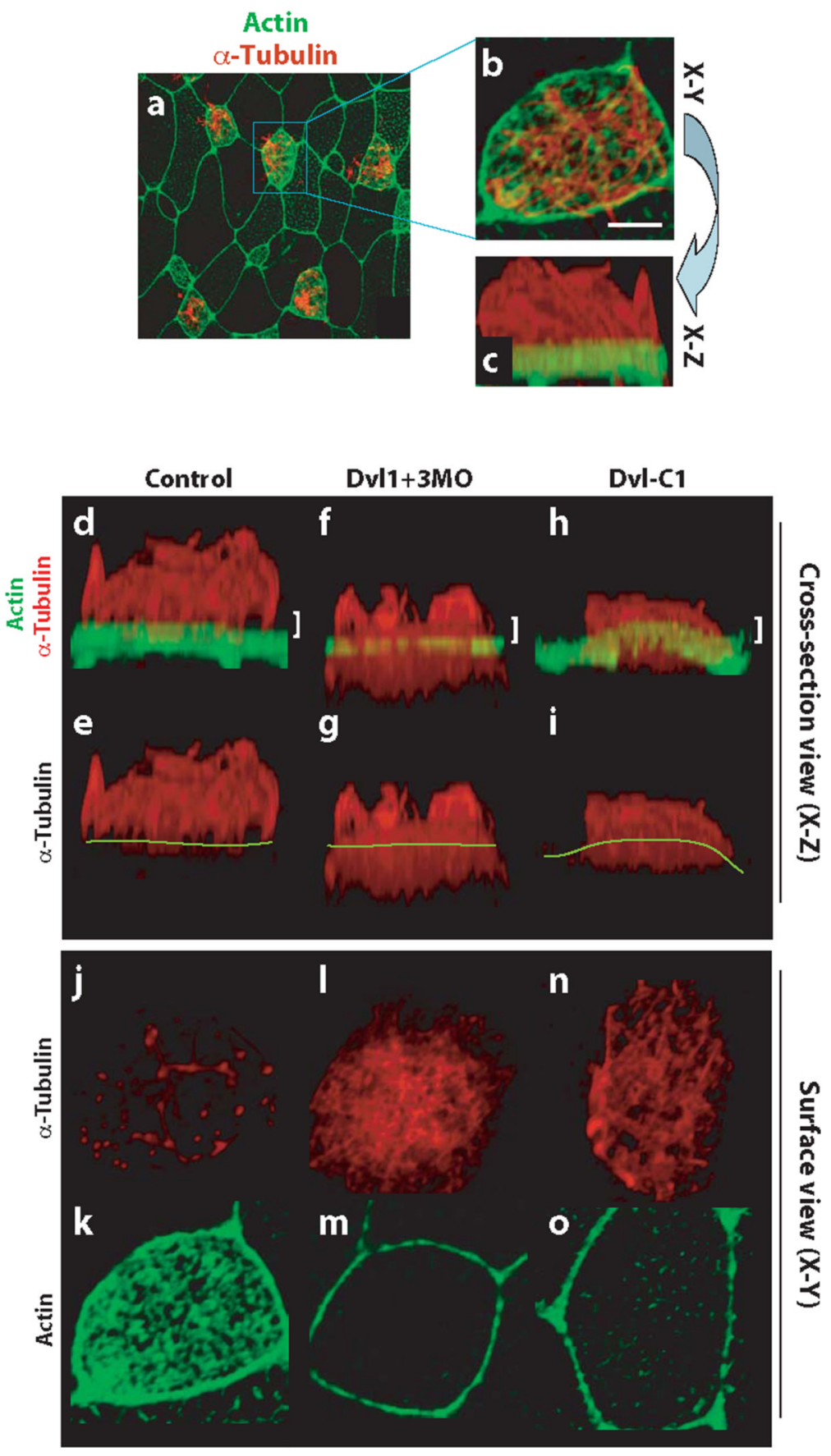

Figure 2. Dishevelled is essential for normal cytoskeletal organization in multi-ciliated cells A. Surface view of a 3D confocal dataset showing the ciliated epidermis of an intact Xenopus embryo. B. At higher magnification, cilia (red, $\alpha$-tubulin) and an apical actin network (green, phalloidin) are visible. Scale bar $=5 \mu \mathrm{m}$. C. Rotation of the 3D confocal dataset shown in B allows the same cell to be viewed in cross-section. D. In a control cell, axonemal microtubules are well organized and form atop dense apical actin filaments. d'. Same image as panel D, but without the green channel; yellow line indicates the apical cell surface. $\mathbf{E}$ and e'. Simultaneous knockdown of Dvl1 and Dvl3 disrupts ciliogenesis and results in microtubule accumulation below or at the apical surface. F and $\mathbf{f}^{\prime}$. Expression of Dvl-C1 disrupts ciliogenesis and results in microtubule accumulation below or at the apical surface. G. Thin 
confocal section at the apical surface (indicated by white bracket in D) reveals that ciliary microtubules project perpendicularly from the cell surface. $\mathbf{g}^{\prime}$. Actin filaments are highly enriched at the apical cell surface. $\mathbf{H}$. Thin confocal section of a Dvl1/Dvl3 double morphant (indicated by white bracket in E) reveals that ciliary microtubules are interwoven parallel to the apical cell surface. $\mathbf{h}^{\prime}$. Apical actin filaments are lost at the surface of morphant cells, but remain at cell-cell boundaries. I. Thin confocal section of anembryo expressing Dvl-C1 (indicated by white bracket in F) reveals that ciliary microtubules are interwoven parallel to the apical cell surface. i'. Apical actin filaments are lost at the surface of DVl-C1 expressing cells, but remain at cell-cell boundaries. 

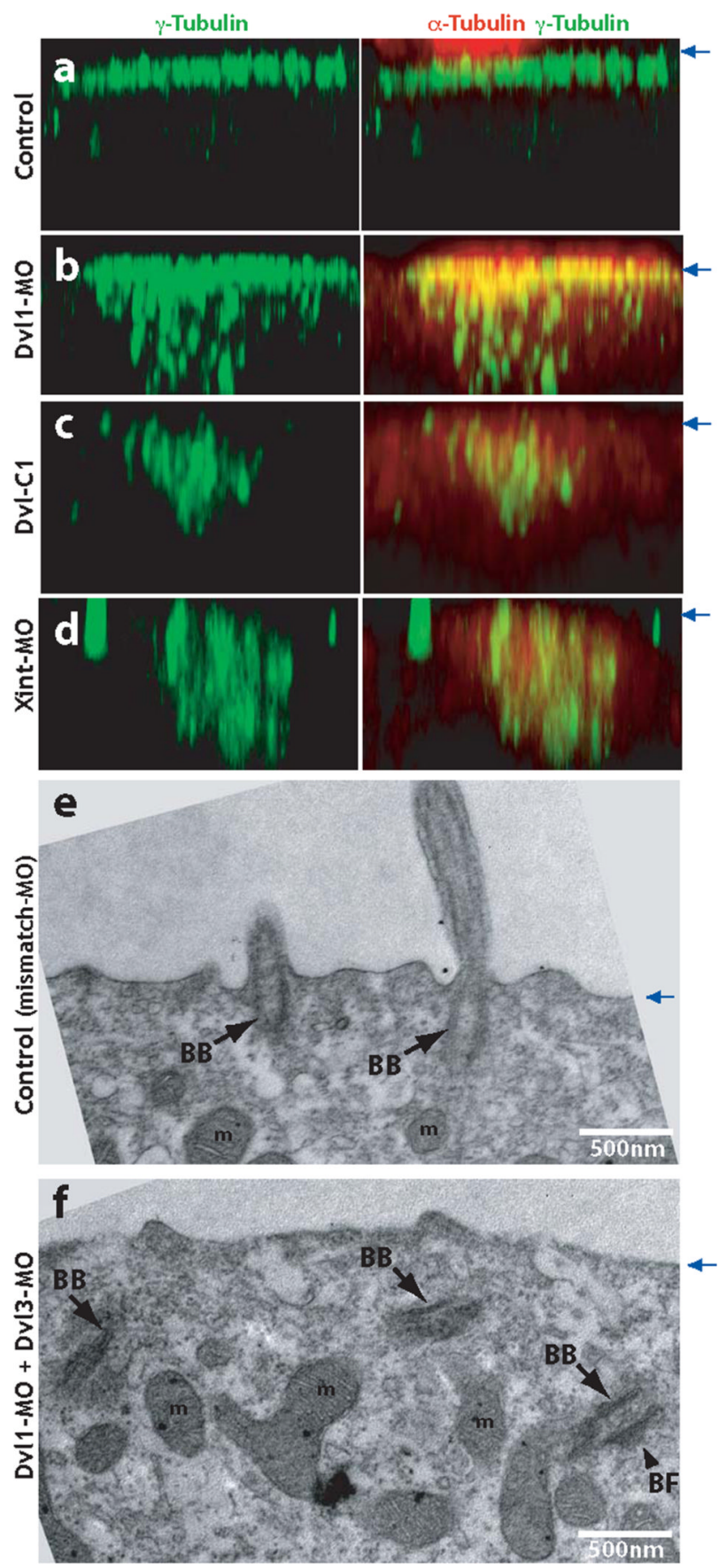

Figure. 3. Dishevelled and Inturned are essential for the apical positioning of basal bodies

A. Control embryos were stained with anti- $\gamma$-tubulin antibody to visualize basal bodies (green). Ciliary microtubules are visualized with anti- $\alpha$-tubulin antibody (red). Serial confocal images are projected in $\mathrm{X}-\mathrm{Z}$ plane, with the position of apical membrane indicated by blue arrows at right. B. Failure of apical basal body localization in Dv11 morphants. C. Failure of apical basal body localization in Dvl-C1 expressing cells. D. Failure of apical basal body localization in Inturned morphants. E. TEM transverse section of control (Dvl1 mismatch MO injected) embryo reveals normal outgrowth of ciliary axonemes from basal bodies docked at the apical cell surface. Cilia can be observed in cross-section projecting above the apical surface. F. Basal 
bodies fail to dock at the apical membrane and remian in the cytoplasm in Dvl morphants. (BB: basal body, BF: basal foot, m: mitochondria). 
Dvl2

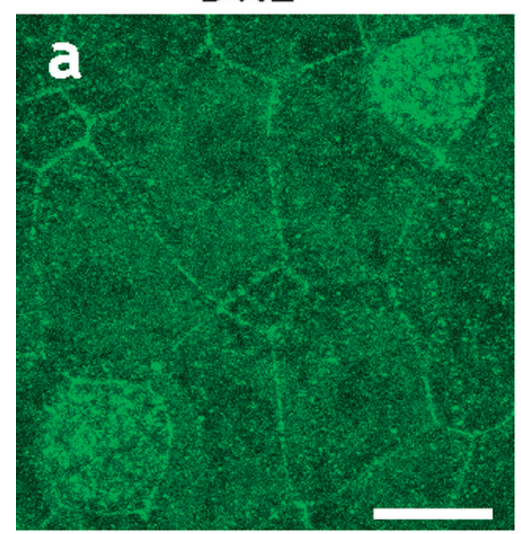

Dvl2

Centrin2-GFP

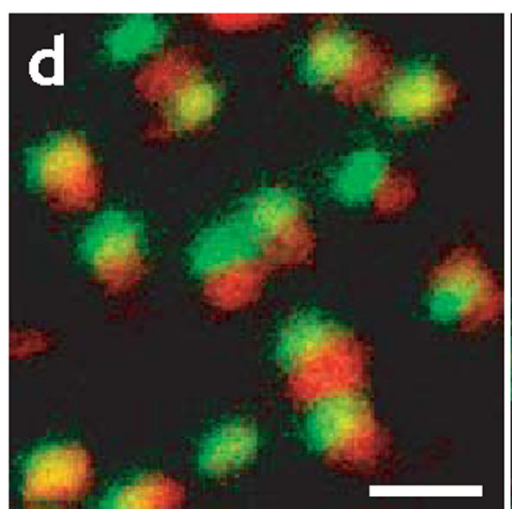

Centrin

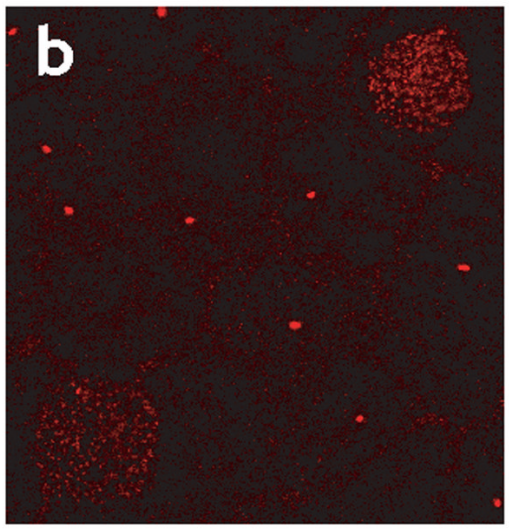

Dvl2 CLAMP-GFP

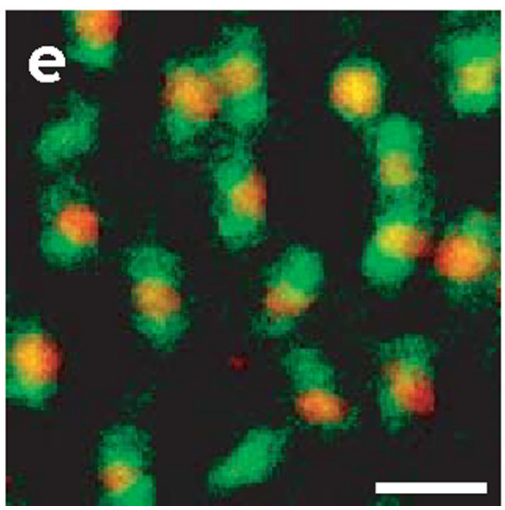

Dvl2 Centrin

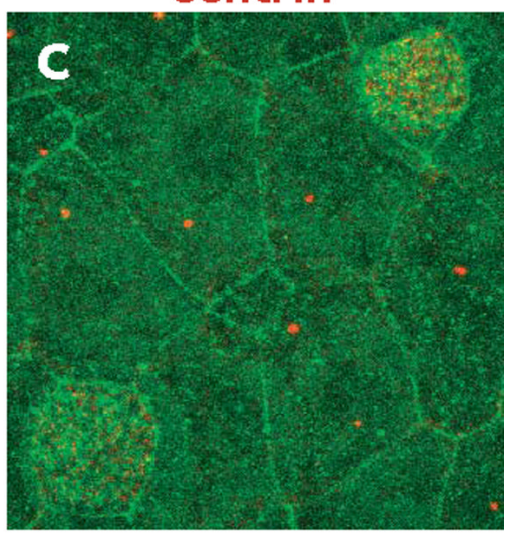

DVI2-C1-GFP

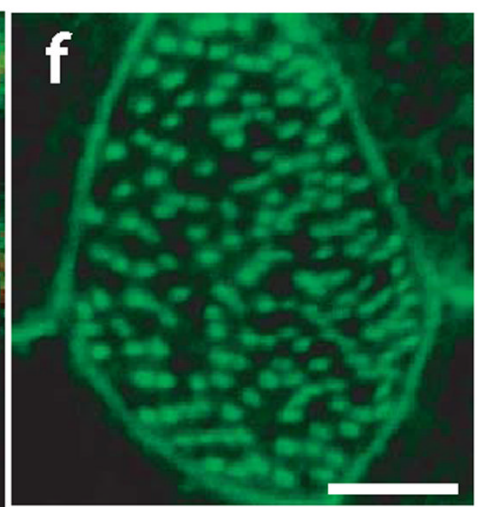

Figure. 4. Dishevelled localizes near the base of cilia in multi-ciliated cells

A. Dvl2 immunostaining reveals enrichment at cell membranes throughout the epidermis and enrichment at the apical surface of ciliated cells, as indicated by immunostaining for centrin (a', a”). Scale bar $=15 \mu \mathrm{m}$. B. Signal for Dvl2 immunostaining (red) localizes immediately adjacent to the basal body, as indicated by Centrin2-GFP (green). Scale bar $=1 \mu \mathrm{m}$. C. Signal for Dvl2 immunostaining (red) localizes to the center of the region marked by CLAMP-GFP (green); data in Supp. Fig. 4 indicate that CLAMP-GFP labels the ciliary rootlet. Scale bar = $1 \mu \mathrm{m}$. D. The C-terminus of Dvl2 is sufficient to drive localization to basal body in multi-ciliated cells. Stage 27 embryos are shown throughout. Scale bar $=5 \mu \mathrm{m}$. 

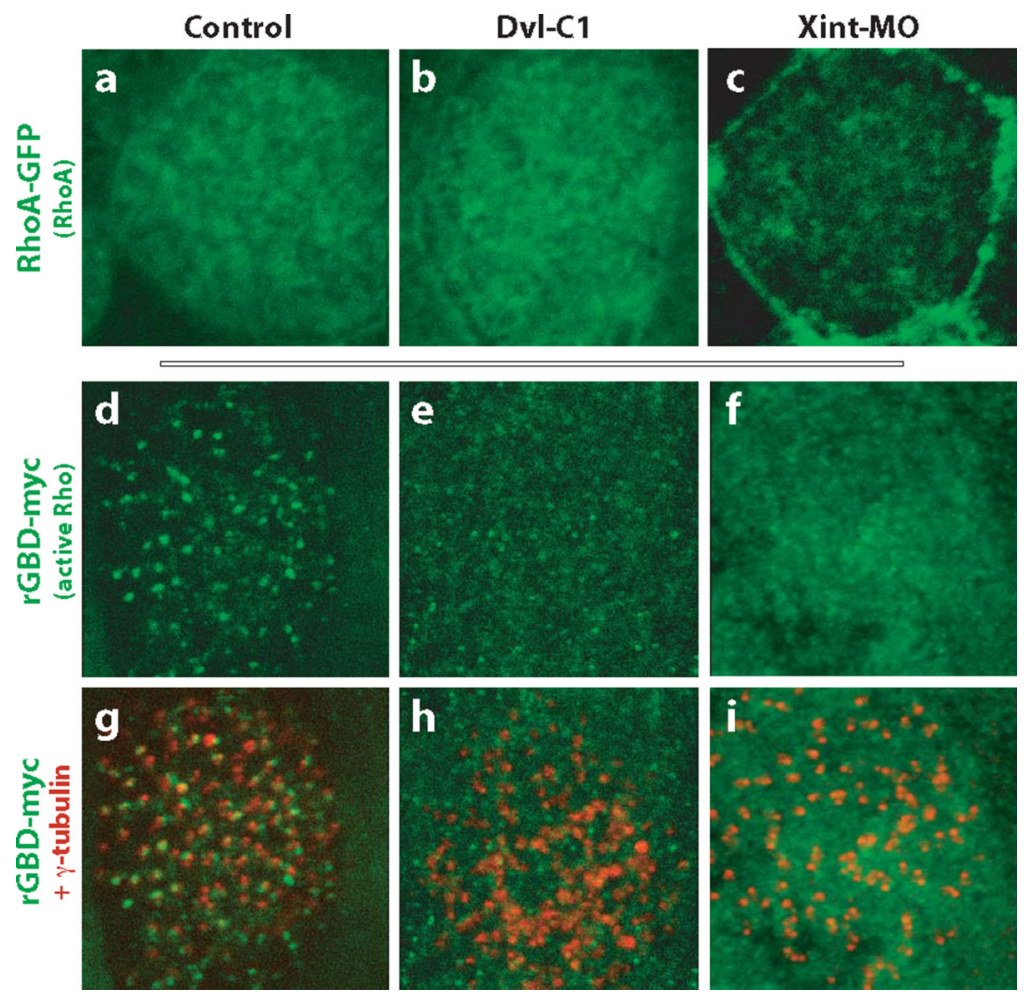

Figure. 5. Dvl and Inturned control the activation and localization of the Rho GTPase

A. RhoA-GFP is enriched apically in control ciliated cells. B. Apical enrichment of RhoAGFP is normal in ciliated cells expressing Dvl-C1. C. Apical enrichment of RhoA-GFP is lost in Inturned morphant cells, while enrichment at cell-cell boundaries was not affected. D. In control cells, the active Rho sensor, rGBD-GFP, specifically localizes near the base of cilia, where $\alpha$-tubulin (red in $\mathrm{d}^{\prime}$ ) appears as puncta due to the perpendicular projection of ciliary axonemes. E. Disruption of Dvl function signficantly reduces the number of rGBD-GFP foci, but does not affect signal at cell-cell junctions. Loss of rGBD-GFP foci correlates with accumulation of interwoven microtubules (red in e') parallel to and below the apical cell surface. F. Disruption of Inturned function signficantly reduces the number of rGBD-GFP foci, but does not affect signal at cell-cell junctions. Loss of rGBD-GFP foci correlates with accumulation of interwoven microtubules (red in $\mathrm{f}^{\prime}$ ) parallel to and below the apical cell surface. 


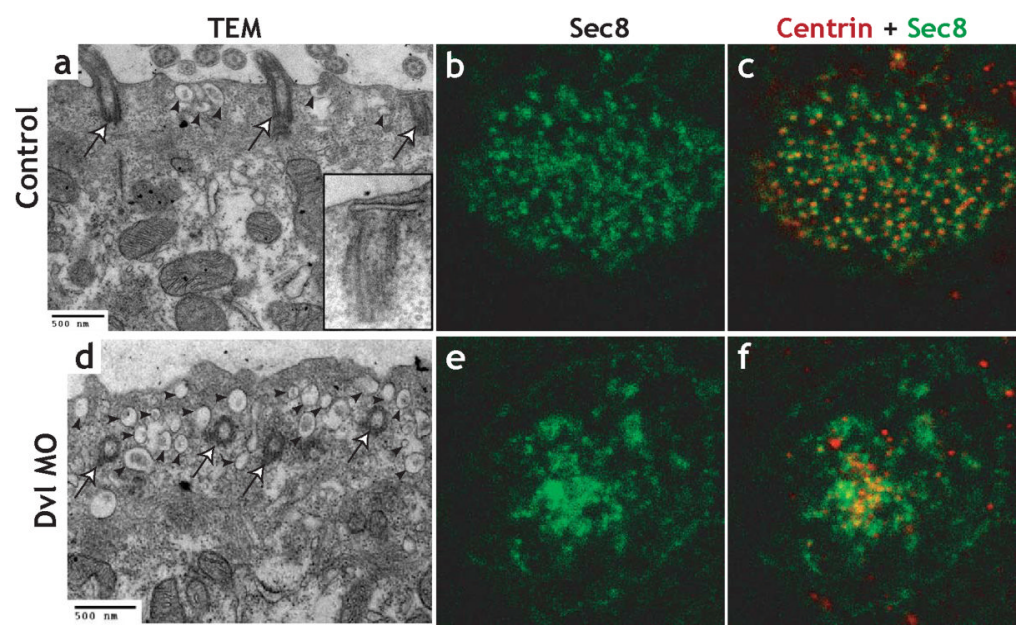

Figure 6. Dishevelled links basal body docking to vesicle traffic and Sec8 localization A. TEM of control embryo. Basal bodies are docked and apical cytoplasm contains very few vesicles (arrowheads). B. TEM of Dvl morphant; basal bodies have failed to dock apically, and the apical cytoplasm contains numerous vesicles (arrowheads). C. Immunostaining reveals an orderly distribution of Sec8 in the apical surface of control multi-ciliated cells. c'. The Sec8 signal (green) associates evenly with basal bodies as indicated by Centrin immunostaining (red). D. Sec8 immunostaining is disordered in Dvl morphants. d'. The Sec8 signal (green) fails to associate with basal bodies (red) in Dvl morphants. 

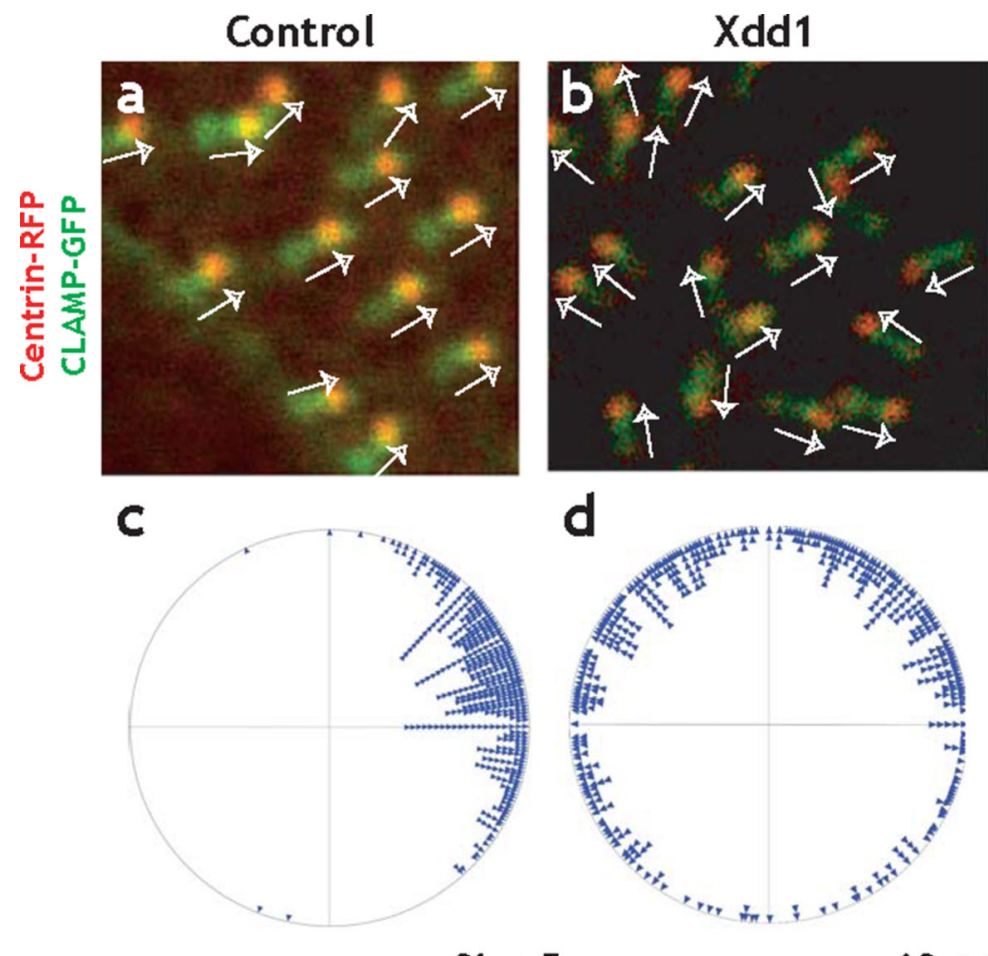

Circular standard deviation $=21+/-5$

$62+/-12$

Figure. 7. Dishevelled governs planar polarization of basal bodies

A. Centrin-RFP and CLAMP-GFP localize to basal bodies and rootlets, respectively revealing the polarized morphology of the basal body complex. In control cells, basal body complexes align parallel to one another (white arrows). B. Polarization can be quantified by angular measurement of individual basal bodies. On the circular plot, each point preresents a single basal body. The circular standard deviation of these measurments is small (21), and is comparable to data obtained using TEM (See Supp. Fig. 4 and Ref. 16). C. Expression of Xdd1 severely disrupts the parallel alignment of basal bodies. D. A circular plot of the angles shows that basal bodies are pointing random directions in Xdd1 expressing cells; the circular standard deviation of these measurments is much larger than controls (62). 

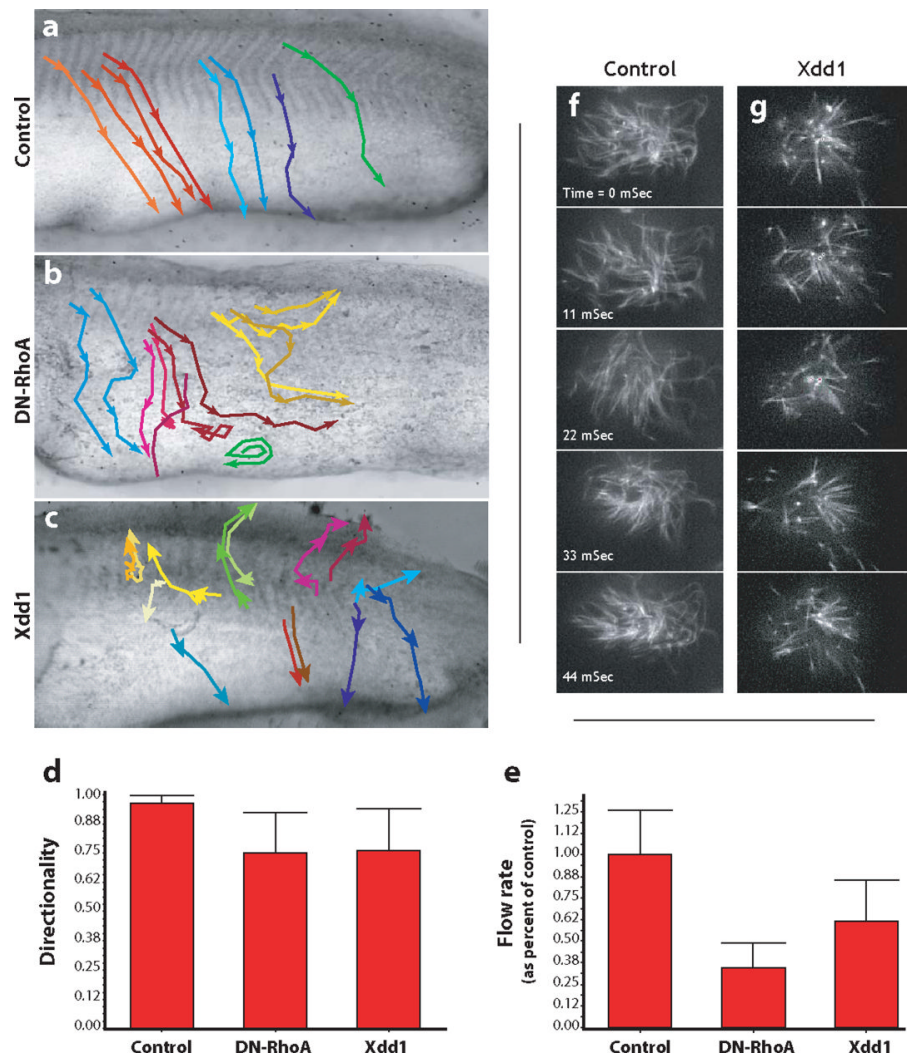

Figure. 8. Dvl and Rho govern directional fluid flow across the ciliated epidermis

A. Control embryos were grown until St. 26 and latex bead were added to the media to visualize fluid flow over the epidermis. The movement of beads was recorded and representative beads were tracked. Representative bead movements were tracked from the horizontal myoseptum, as indicated by the colored lines. Beads move from dorso-anterior to ventral-posterior uniformly in control embryos. B. DN-RhoA expression severely disrupts directional flow; beads were turning and swirling frequently. C. Xdd1 expression severely disrupts directional flow; beads were turning and swirling frequently. D. The directionality of beads was quantified as the ratio of the linear distance between the first and last point of a track and the total diatance along the track. A straight line would be a value of 1.00. E. DN-RhoA or Xdd1 reduces the rate of bead movement across the epidermis. Finally, defects in polarized flow were evident in that even beads moving in comparatively straight paths moved at a greatly reduced rate across experimental embryos. F. Stills from a high-speed time lapse movie of beating cilia labelled with tau-GFP. G. Stills from a high-speed time lapse movie of beating cilia expressing $\mathrm{Xdd} 1$, frequency is comparable to control. 